\title{
Development and Evaluation of a Smartphone Application for Self-estimation of Daily Mental Stress Level
}

\author{
Shuwa KIDO, Ayako HASHIZUME, Tetsuaki BABA and Takemi MATSUI
}

Tokyo Metropolitan University, 6-6 Asahigaoka, Hino, Tokyo 191-0065, Japan

\begin{abstract}
Heart rate variability (HRV) is known to represent the activity of the autonomic nervous system and is used as a measure for stress levels. Generally, HRV is calculated from the heart rate or the pulse wave using an electrocardiogram (ECG) that is physically-taxing. However, for those with psychological strains and disorders, a handy device that can be used at any time is necessary in order to detect mental illness in its early stages and to prevent it from becoming worse. Therefore, we focused on the idea of using a popular device, the smartphone, and we developed a real-time, HRV-calculating application that uses a photoplethysmogram (PPG) obtained with the smartphone's camera. The user-friendly interface of the application was designed for use by a wide range of age groups. We conducted two experiments and compared the HRV data obtained by our application with ECG measurements to verify the application's accuracy. Once a certain level of accuracy was reached, we evaluated the usability of our application among young and elderly users. The results showed that improvements are necessary in terms of the interface, measuring time, and other performance indices.
\end{abstract}

Keywords: HRV, smartphone, non-invasive measurement, usability

\section{INTRODUCTION}

According to a survey conducted by the Ministry of Health, Labour and Welfare (MHLW, Japan) in 2012, the number of patients with depression, schizophrenia, and anxiety disorders has increased year by year [1]. These mental diseases are frequently caused by psychological strains and burdens. It is thought that self-measurement of daily stress levels will make it possible to detect these mental illness in the early stages.

There are subjective and objective methods for grasping mental stress levels. Subjective methods, such as the questionnaire, are very useful for assimilating psychological transitions in the subject. However, results of these methods are easily influenced by subjects' retention of the evaluation criteria. If we adopt these methods on a daily basis, the results may not be continuous but discrete. In other words, subjective methods are not sufficient for monitoring the daily mental stress.

Whereas, there are objective methods of using indicators such as the heart rate variability (HRV) [2] and the salivary amylase level. These objective methods can estimate mental stress levels more reliably than subjective methods. However, these objective methods are not suitable for daily use because they require special devices, technical skills, and fundamental knowledge. For example, HRV is calculated from the heart rate or the pulse wave using electrocardiogram (ECG) or photoplethysmogram (PPG) that require special measurement devices such as electrocardiographic monitors and sphygmographs. When calculating mental stress levels for the early detection and prevention of mental illness, daily measurements are essential. Furthermore, an objective method with the simple equipment that enables easy measurement is desirable.

As mentioned above, we developed an application that can estimate mental stress levels more easily than the conventional method such as the ECG. For assessing daily levels of mental stress, the objective method is superior to the subjective method but requires special devices. In order to solve this problem, we developed a special-device-free estimation of mental stress levels.

On the other hand, the progress of aging is very rapid in Japan and since 2005 Japan has become the most aged society in the world. The percentage of elderly people (percentage of total population aged 65 and over) in Japan has risen up from 5\% in 1950, 10\% in 1985, and $20 \%$ in 2005 , to $25 \%$ in 2013 , and is expected to rise to $30.3 \%$ in 2025 and $39.9 \%$ in 2060 [3]. Against these factors, manufacturers in electronics have developed cell phones, smartphones and computers for elderly users $[4,5]$, but there still exists a gap between high-skilled end users and low-skilled end users depending on their demographic traits. One of the marked differences 
is related to differences within the age group [6]. The usability of the device is significant to the elderly, so the usability of software such as smartphone applications should also be considered through the spread of smartphones.

Therefore, we performed an experiment to verify the application's precision and conducted a user evaluation of the application. The experiment was conducted to compare HRV results from the proposed method with those from the conventionally used ECG. The evaluation focuses particularly on assessing usability of the application among young and elderly users.

\section{THE SMARTPHONE HRV CALCULATION ALGORITHM}

The HRV is used as a mental stress indicator in various studies. Generally, it is calculated from the heart rate or the pulse wave using ECG or PPG that requires special measurement devices, technical skills, and knowledge. Figure 1 shows the flow of calculation of HRV in the conventional method using many devices for measuring ECG. It also shows the proposed method to measure the PPG using only a smartphone.

Figure 1 presents the measurement method for the electrocardiogram, affixing electrodes to the surface of the human body. Electrodes are connected to the electrocardiograph to measure the potential difference due to heart movements as detected on the surface of the body in the conventional method. The analog data is sent to the PC through an A/D converter. The heart rate fluctuation data obtained from the electrocardiographic wave is sent to the PC and the HRV will be calculated using frequency analysis. We developed an application that can calculate the HRV more easily than the conventional method. The calculation algorithm adopted a white light-emitting diode (LED) light of the smartphone as a light source and smartphone CMOS camera as a photo sensor by applying the pulse wave sensing

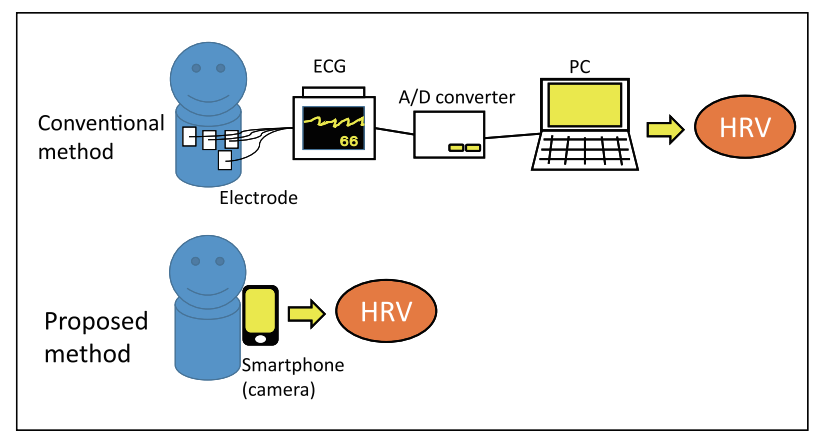

Figure 1: HRV calculation flow

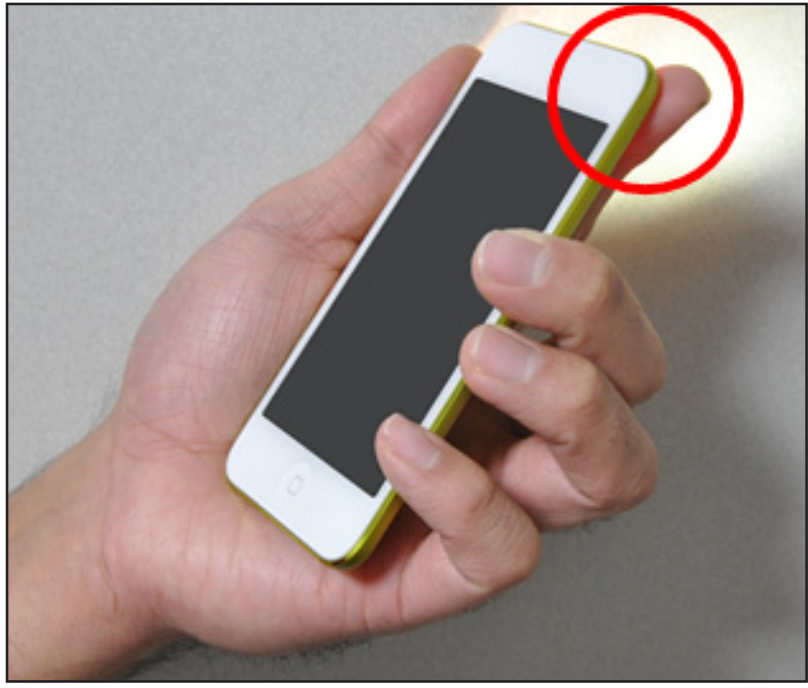

Figure 2: Measurement of the pulse wave with the smartphone application

technology [7]. By means of the low sampling rate of smartphone $(33 \mathrm{~Hz})$, we determined PPG and corresponding time-series heart beat intervals.

Figure 2 shows how to hold the smartphone during the HRV measurement using our application. The first step is to place a fingertip over the lens of the smartphone camera and the LED. The left index finger is preferable. The camera acquires images of blood vessels in the fingertip, and obtains PPG data by measuring the timing of the blood flow. Using the above algorithm, we can calculate HRV from the PPG data using a smartphone.

\section{EXPERIMENTAL VERIFICATION OF PRECI- SION}

\subsection{Method}

In order to verify the accuracy of the HRV calculation, we compared the HRV measured by our application with that obtained from an ECG and examined whether there was a correlation between the results of these two methods. We conducted the HRV measurement with ten young people in their 20s $(22.1 \pm 0.8$ years of age $)$. During measurement, the participant was seated in a chair and was instructed to tap a finger on the camera lens and the LED light of the smartphone to obtain stable data. This measurement continued for 150 seconds.

Our data processing program detected the peaks of the PPG. In order to calculate the HRV, the RRI fluctuation wave was interpolated and re-sampled at intervals of $250 \mathrm{msec}$. The power spectrum of the re-sampled RRI was calculated using fast Fourier transform (FFT). By 
adopting FFT to obtain HRV, we were able to calculated HRV from 64 seconds RRI.

There are many indices related to HRV. For example, the low-frequency index (LF) represents autonomous nervous activity, the high-frequency index (HF) represents parasympathetic nervous activity, and the ratio of LF to $\mathrm{HF}(\mathrm{LF} / \mathrm{HF})$ represents sympathetic nerve activity. We adopted $\mathrm{LF} / \mathrm{HF}$ as a comparative index because it has often been used to represent the mental stress level in previous studies.

\subsection{Results}

Figure 3 illustrates the R-R interval (RRI) obtained from both methods. According to the verification experiment, we confirmed that the two fluctuation waves were almost the same. The average and minimum correlations among all subjects were $r_{\text {average }}=0.90$ and $r_{\text {minimum }}=0.81$, respectively. LF/HF also showed a strong positive correlation $\left(r_{\mathrm{LF} / \mathrm{HF}}=0.96\right)$ between the two methods (Figure 4).

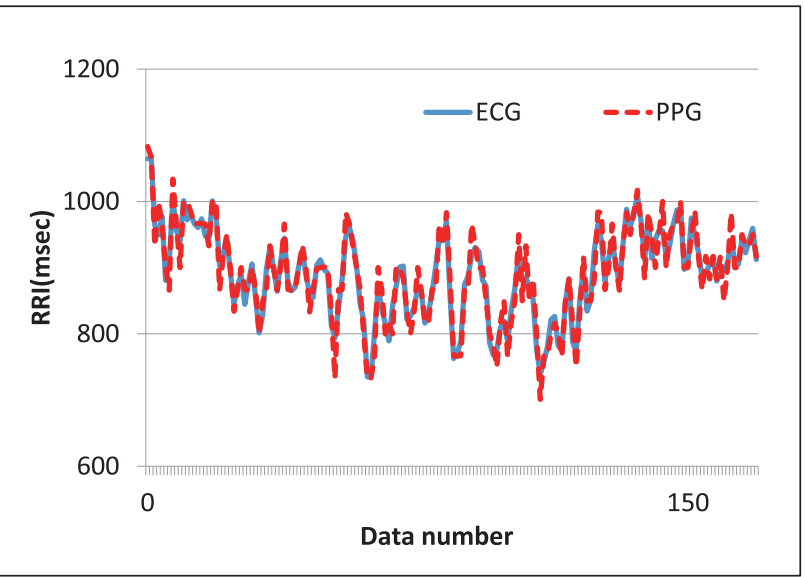

Figure 3: Fluctuation waves of both ECG and PPG methods

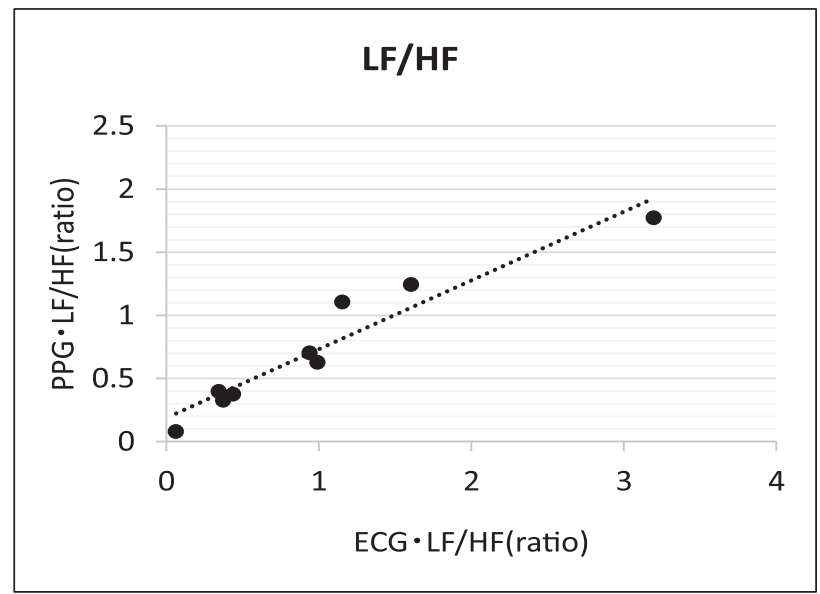

Figure 4: Correlation coefficients of LF/HF

\section{USER EVALUATION}

We conducted a usability evaluation for the purpose of getting detailed information about the users' subjective impressions and requirements for the application.

\subsection{Method}

Participants included 19 young people in their 20s and 6 elderly people in their 60-70s, all living in Tokyo. The average age of the young people was 21.5 years with a standard deviation (SD) of 1.5 years, and the average age of the elderly users was 69.3 years with an SD of 2.4 years. All 19 young participants had their own smartphones and all 6 elderly people had cell phones.

We defined elderly people as those who are over 65 years of age, based on the World Health Organization (WHO) definition.

First we explained the research it is for the academic purposes and does not have anything to do with the sales or marketing. We then told the participants the expected duration of the experiment and that they were free to quit at anytime during the experiment should they feel uncomfortable with our questions or our treatment of any information gathered.

After obtaining their consent, we explained the evaluation procedure and how to use the application to each participant. Then the participants performed a trial to measure their mental stress level using the application. After the measurement, subjects answered the following questions and commented on the application.

The user evaluation included the following indicators:

1. Demographic characteristics (name, gender, age, experience with using ECG, etc.),

2. Number of errors during the measurement,

3. Whether or not the measurement was successful, and

4. Impressions after using the application.

Only the results of the usability evaluation and the length of measuring time required are described in this paper. Usability and time required for measurement were evaluated with scores from 1 to 5 . Along with this evaluation, free-form comments on our application were collected from the subjects, leading to successful extraction of users' requirements.

\subsection{Results}

The results of the evaluations are summarized in Figures 5 and 6. The mean scores on usability evaluations were 3.8 for the young group and 3.7 for the elderly group. Little difference was obtained between the two groups (unpaired t-test, $\mathrm{df}=23, \mathrm{t}=-0.25, \mathrm{P}=$ n.s.; Fig. 5). 


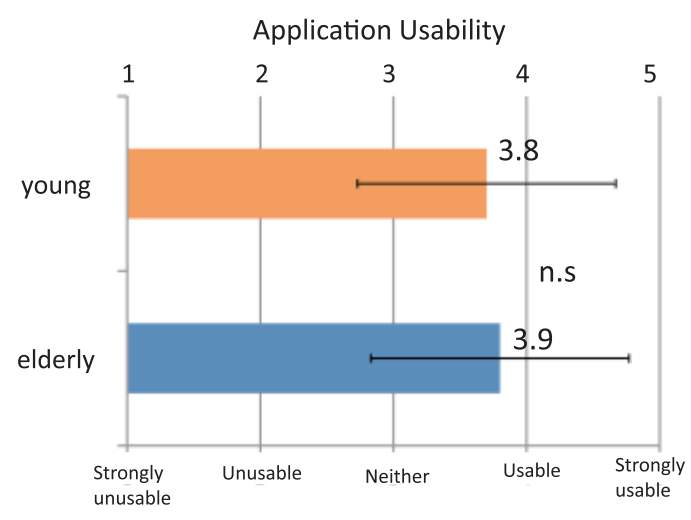

Figure 5: Results of usability evaluation

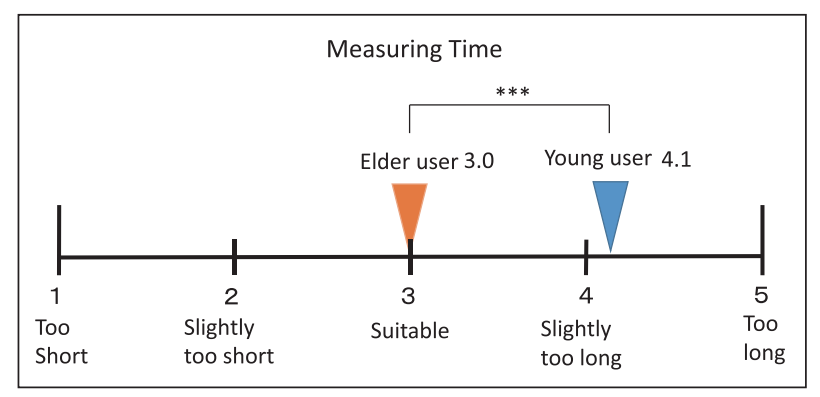

Figure 6: Result of evaluation of required measurement time

The mean scores for the time required for measurement was 4.1 and 3.0 for the young and elderly groups, respectively (unpaired t-test, $\mathrm{df}=23, \mathrm{t}=-4.06, \mathrm{P}<0.01$ Fig. 6). Many subjects in the young group reported that the measurement took too long compared with the reports from the elderly group. Furthermore, suggestions for the application were obtained, such as "Measurement time was too long" (young user) and "It was difficult to hold the smartphone" (elderly user).

\section{DISCUSSION}

According to the verification experiment, HRV measurement using our application was found to be valid for assessing mental stress with the same precision as the ECG method.

The application was confirmed to be easy to use, regardless of the age group. The group of young users found the measurement time to be long. All the young group members were using smartphones in their daily lives, hence they are accustomed to using such applications. Because the application does not require any special operation, they reported boredom while staring at the screen for 64 seconds. On the other hand, several members of the group of elderly users who had experience of ECG measurement in hospitals found the measurement time to be short.

\section{CONCLUSION}

We have developed an application that can calculate the mental stress level using an objective method and can be used in daily life. We used the smartphone as the measurement equipment because of its convenience. We developed an application that can estimate mental stress levels more easily than the conventional method using an ECG.

We confirmed the accuracy of HRV detected with our application and its usability with a verification experiment and a user evaluation, respectively. The result of the comparative experiment indicated a strong positive correlation between HRV calculated from the ECG and from the smartphone. The results of the user evaluation showed that the proposed application is easy to use regardless of age and prior smartphone experience. Although several young users felt that the measurement time was too long, measurement time cannot be shortened because of possible adverse influence on HRV calculation accuracy.

These results showed that our application can measure mental stress levels with the same accuracy as the conventional method and that our application was appropriate for everyday use. So far, the required measurement time has been the main problem we have faced; this is to be solved by improving the application interface so that users remain interested while taking measurements.

\section{REFERENCES}

1. Ministry of Health, Labour and Welfare; Patient Survey, 2011.

2. Toshikazu Shinba; Altered autonomic activity and reactivity in depression revealed by heart rate variability measurement during rest and task conditions, Psychiatry and Clinical Neurosciences, 68, pp.225233, 2014.

3. Cabinet Office Japan; Annual Report on the Aging Society, 2014.

4. Ministry of Internal Affairs and Communications; A Report on the Use of Communication System in 2013, 2014.

5. Toru Irie, Keigo Matsunaga and Yukinori Nagano; The Challenge to the Universal Design in the Development of the Cell Phone "Easy Phone (Raku-Raku Phone)", FUJITSU, 56(2), pp.146-152, 2005.

6. Kenetsu Furuki and Yoshiaki Kikuchi; Approach to Commercialization of RakuRaku Smartphone, FUJITSU, 63(5), pp.548-554, 2012. 
7. Christopher G. Scully, Jinseok Lee, Joseph Meyer, Alexander M. Gorbach, Domhnull Granquist-Fraser, Yitzhak Mendelson and Ki H. Chon; Physiological Parameter Monitoring from Optical Recordings with a Mobile Phone, Biomedical Engineering, IEEE Transactions, 59(2), pp.303-306, 2012.

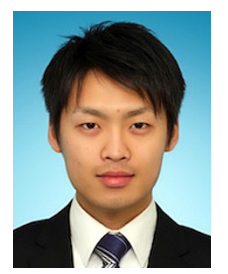

\section{Shuwa KIDO}

Shuwa Kido is a master course student of Graduate School of Tokyo Metropolitan University, Japan. He graduated from Tokyo Metropolitan University in 2014. He has been researching biomedical engineering and HCD (human centered design). He is developing new medical systems using vital signs, such as heat rate, pulse wave and respiration. His representative work of "UTSUKAMO" is a smart phone application that can provide a method for examining the depression by which whether a user is suffering from the depression or not, can simply be determined by using user's HRV (heart rate variability). He developed the HRV calculation algorithm and designed the interface of "UTSUKAMO" based on the HCD process. He was awarded the best presentation prize at the winter research meeting 2014 from the HCD-Net (Human Centered Design Network).

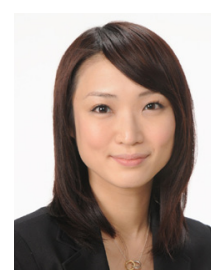

\section{Ayako HASHIZUME (Member)}

Ayako Hashizume received Ph.D. in Kansei Science from Tsukuba University, Japan, in 2011. She is an assistant professor at the Tokyo Metropolitan University. Her research interests include psychology of emotion and Kansei, engineering in relation to HCD (human centered design) and UX (user experience), especially among elderly users. She was awarded the kids design prospective competition 2008 from the Japan Kids Design Association, the best design prize at the design marathon 2009 from the International Association for Universal Design and the research prize 2013 from the Funai Foundation for Information Technology. She is a chief of the special interest group on Kansei of HCD-Net (Human Centered Design Network). She is also a member of Human Interface Society (Japan) and Society of Socio-Informatics (Japan).

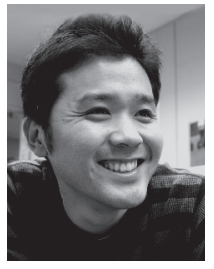

\section{Tetsuaki BABA}

His research focuses on Interaction Design and Media Art. His works and researches, such as musical instruments, experimental devices, gadgets, educational materials and so on, are located in the area of fusion of product design and media art. He received Ph.D. degrees in art and technology from Kyushu University, Japan, in 2009. He is an associate professor at Tokyo Metropolitan University, Tokyo, Japan. One of his work "Freqtric Drums" is a device that turns users into musical instruments so that users can not only play the instruments but also communicate with the others by touching each other. Through digital technology, Freqtric Drums allows participants to simultaneously enjoy music and skin contact communication and is corrected by art/science centre all over the world as a permanent correction. He is the member of ACM, IPSJ, ADADA, VRSJ.

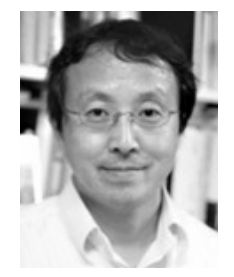

Takemi MATSUI

Takemi Matsui received the B.S. and M.S. degree from the University of Tsukuba in physics in 1984 and 1986 respectively. He received the Ph.D. degree in medicine in 1995 from Saitama Medical School.

He proposes a variety of screening systems, including vital signs based non-contact infection screening system, and depression screening system by autonomic nerves monitoring. He joined National Defense Medical College as a faculty member in 1997, and has been a professor of medical engineering of Tokyo Metropolitan University since 2005. 